\title{
Análisis del comportamiento productivo de cerdas al parto en granja semiintensiva
}

\section{Analysis of the productive behavior of sows at delivery in semi-intensive farm}

\author{
SANCHEZ-CHIPRES, David Roman †*, MORENO-LLAMAS, Gabriel, JIMÉNEZ-PLASCENCIA, \\ Cecilia y JIMÉNEZ-CORDERO, Ángel Andrés
}

Universidad de Guadalajara, Departamento de Producción Animal CUCBA

ID $1^{\text {er }}$ Autor: David Roman, Sanchez-Chipres / ORC ID: 0000-0002-5273-0393, CVU CONACYT ID: 69431

ID $1^{\text {er }}$ Coautor: Gabriel, Moreno-Llamas / ORC ID: 0000-0002-1003-1738, CVU CONACYT ID: 101392

ID $2^{\text {do }}$ Coautor: Cecilia, Jiménez-Plascencia / ORC ID: 0000-0003-1705-792X, CVU CONACYT ID: 1000339

ID $3^{\text {er }}$ Coautor: Ángel Andrés, Jiménez-Cordero / ORC ID: 0000-0002-1734-2678, CVU CONACYT ID: 947963.

\section{Resumen}

El propósito de este trabajo fue evaluar el comportamiento productivo de cerdas en una granja semitecnificada. Se utilizaron cerdas F1 York $\mathrm{x}$ Landrace como línea materna y fueron inseminadas con semen Pietrain como línea paterna. Se consideraron 13 camadas contemporáneas. Se registró tamaño de camada al nacimiento, pesos al nacimiento y al destete, así como lechones destetados y la información se registró en el programa pigchamp (C). Considerando la información de todas las cerdas de este estudio, el tamaño de la camada al nacimiento y del peso al nacimiento pueden considerarse relativamente buenos comparados con los obtenidos en otros estudios. Los resultados en las correlaciones de acuerdo al número de parto de las cerdas, indicaron la existencia de una correlación alta y positiva con todas las variables de estudio, similar al obtenido por Murillo et al. (2007), lo que resalta el uso de estas variables como indicadores de la productividad materna de las cerdas la cual incrementa en cada parto.

Producción, Lechones, Temperatura

\begin{abstract}
The purpose of this work was to evaluate the productive behavior of sows in a semi-technical farm. F1 York x Landrace sows were used as the maternal line and were inseminated with Pietrain semen as the paternal line. 13 contemporary litters were considered.Litter size was recorded at birth, weights at birth and at weaning, as well as weaned piglets and the information was recorded in the pigchamp (C) program. Considering the information of all the sows in this study, the size of the litter at birth and the weight at birth can be considered relatively good compared to those obtained in other studies. The results in the correlations according to the number of sows birth, indicated the existence of a high and positive correlation with all the study variables, similar to that obtained by Murillo et al. (2007), which highlights the use of these variables as indicators of the maternal productivity of sows, which increases in each birth.
\end{abstract}

Production, Piglets, Temperatura

Citación: SANCHEZ-CHIPRES, David Roman, MORENO-LLAMAS, Gabriel, JIMÉNEZ-PLASCENCIA, Cecilia y JIMÉNEZ-CORDERO, Ángel Andrés. Análisis del comportamiento productivo de cerdas al parto en granja semiintensiva. Revista de Innovación Sistemática. 2019. 3-9: 12-17

\footnotetext{
$\dagger$ Investigador contribuyendo como primer Autor.
} 


\section{Introducción}

El consumo de carne de cerdo en México en los últimos diez años creció a una tasa promedio anual de 3.9 por ciento, al pasar de 1.4 millones de toneladas en 2006 a 2.1 millones de toneladas en 2018. Se prevé que se mantenga esa tendencia durante 2019, alcanzando un consumo de 2.2 millones de toneladas de carne, un crecimiento anual de 4.3 por ciento. Entre 2014 y 2018, el 69 por ciento del consumo de carne de cerdo en México provino de la producción nacional, mientras que el 31 por ciento se abasteció de importaciones. La carne de cerdo es una alternativa de menor costo en comparación con la carne de bovino, y su precio es competitivo con la carne de ave.

Jalisco es un estado eminentemente porcícola, ubicando su producción durante los últimos treinta años entre las tres primeras del país, siendo su producción en el 2018 de 321,735 toneladas de carne (SAGARPA-siap 2019). Se reconocen cuatro regiones especializadas en la producción: región altos norte (con el municipio de Lagos de Moreno como cabecera regional); región altos sur (Tepatitlán); región sur (Ciudad Guzmán) y región centro (Zapopan), manifestándose como las principales zonas productivas las regiones altos norte y sur con un $85.6 \%$ de la población total calculada en un 3,413,513 cerdos (INEGI 2016).

La producción porcina de la región jalisciense está encaminada a la satisfacción social de proteína de origen animal, su organización es desde un enfoque de la teoría de sistemas clasificada en tres tipos generales: sistemas de producción animal intensivos, semi intensivos, y extensivos. Esta clasificación está definida por los niveles de uso de tecnologías para la manipulación del medio ambiente y del manejo de las poblaciones animales, las cuales son mucho más densas y de mayor confinamiento en los modelos de producción intensiva. Los sistemas de producción intensiva se organizan y operan bajo la teoría del capital, lo cual implica, mayor trabajo en el menor tiempo posible, ello sólo es factible en la medida que se controle la uniformidad de cada eslabón de la cadena productiva, asegurando también uniformidad del producto a comercializar. Esto provoca la dependencia de altas tecnologías.
El recurso esencial del sistema es el cerdo, el cual para garantizar la dinámica productiva debe proveer una población de individuos con características uniformes. Esto se logra con animales genéticamente homogéneos que se obtienen con altas presiones de selección, para ello se requiere contar con un ambiente estable y de confort; así los niveles de intensidad del sistema de producción animal, intensivo, semi-intensivo o extensivo, se establecen al definir los niveles de control del entorno ambiental (POET,1998).

La producción intensiva ocupa la mayor parte de la producción en Jalisco; ésta se realiza principalmente con híbridos raciales, aunque no todos provengan de esquemas de cruzamientos planificados. Aún más, el pie de cría de diferentes genotipos raciales, proviene de diversos países industrializados, por ejemplo, de Canadá, Estados Unidos o Inglaterra, lugares de donde preferentemente se importan tanto sementales como hembras reproductoras.

Los productores especializados de Jalisco están buscando aquellos cambios tecnológicos que les permita alcanzar en menor tiempo un mayor grado de eficiencia, ya que se pretende obtener cerdos a las 21 semanas de edad con un peso de mercado de $100 \mathrm{a} 110 \mathrm{~kg}$. El mejoramiento de la calidad del alimento y de los sistemas de alimentación son los avances comúnmente utilizados en las granjas tecnificadas.

Los engordadores persiguen un aumento diario de peso de sus cerdos a través del mejoramiento genético, sin embargo, en lo que respecta a éste, en el estado de Jalisco no se tiene un esquema de producción uniforme con relación a los tipos de cruzamientos que se utilizan en la producción de animales para abasto.

Se utilizan como líneas maternas, cerdas $\mathrm{F}_{1}$ : Yorkshire-Landrace, Yorkshire-Hampshire, Large White-Landrace o Landrace- Hampshire y como líneas paternas las razas puras entre, las que sobresalen: Duroc, Hampshire, Pietrain o híbridos de estas razas, (Flores y Gómez, 1995). 
Esto lleva a producir cerdos con diferencias en cuanto a desempeño reproductivo y eficiencia alimenticia, además con diferente capacidad de adaptación a diversos factores ambientales, esto se hace evidente al comparar el desempeño de los cerdos nacionales con respecto a los cerdos de otros países, como, por ejemplo, diferencias de 0.7 de lechón al nacimiento y $0.6 \mathrm{~kg}$ del peso al destete, inferiores en los cerdos nacionales con respecto a los cerdos de Estados Unidos (Batista, 2000).

\section{Planteamiento del problema}

pueden resultar no solamente en un bajo aprovechamiento de la cerda, sino que también limitarán la posibilidad de mejoramiento genético de la piara. Las características reproductivas en general son de baja heredabilidad, por lo que gran parte de la mejoría en estas variables se puede conseguir reforzando las buenas prácticas de manejo, una de estas corresponde a los sistemas de alojamiento los cuales influyen en el comportamiento productivo de los cerdos asi como las condiciones climáticas.

\section{Justificación}

Actualmente las granjas requieren de una mayor productividad para tener eficiencia financiera. El pie de cría representa la base para mantener parámetros productivos por lo que se requiere de condiciones medioambientales que permitan expresar su capacidad reproductiva, ante ello la información y análisis de la misma permitirán identificar áreas de mejora en la granja.

\section{Objetivo general}

Evaluar el comportamiento productivo de cerdas en una granja semitecnificada.

\section{Objetivos Particulares}

Evaluar parámetros de lechones nacidos vivos, peso al nacimiento, lechones destetados y peso al destete. Correlacionar efectos de los parámetros, entre los que se encuentran el tamaño de la camada al nacimiento y al destete, el número de partos por hembra por año, el número de lechones destetados por hembra por año y el número de cerdos vendidos por hembra por año.

\section{Materiales y Métodos}

Localización: El presente estudio se realizó en una granja comercial ubicada en el municipio de Zacoalco de Torres, Jalisco.

La granja es semi tecnificada y cuenta con 230 vientres y es manejada en dos sitios correspondiendo a esta ubicación el sitio uno.

Animales: Se utilizaron cerdas F1 York x Landrace de diferentes partos, como línea materna y fueron inseminadas con semen Pietrain como línea paterna.

Metodología Experimental: Para este trabajo se consideraron 13 camadas contemporáneas.

Se registró tamaño de camada al nacimiento, pesos al nacimiento y al destete, así como lechones destetados y la información se registró en el programa pigchamp®.

\section{Diseño experimental}

Se realizó análisis de regresión con los resultados obtenidos en el experimento.

\section{Resultados}

La tabla 1 muestra la información de las cerdas y sus respectivas camadas del parto al destete, comparada con la temperatura promedio del ambiente en que estuvieron los lechones los primeros siete días.

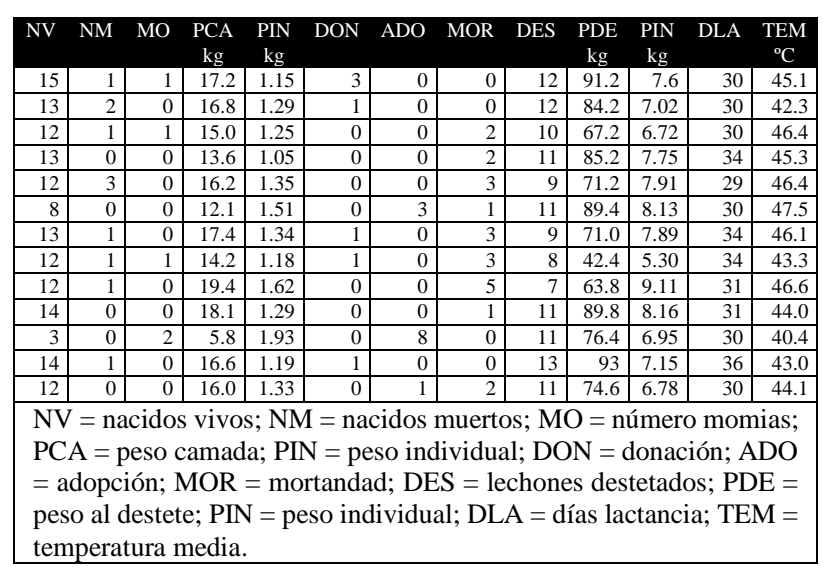

Tabla 1 Registro productivo de 13 cerdas del parto al destete 


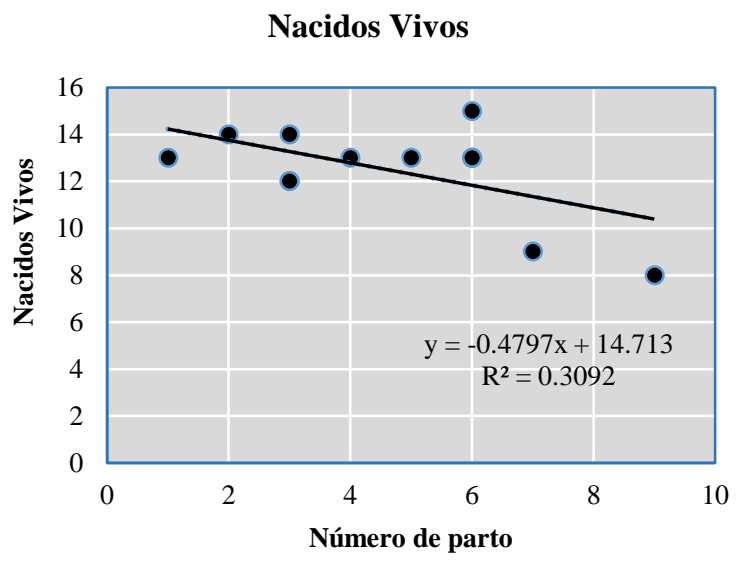

Gráfica 1 Regresión entre número de parto y lechones nacidos vivos

La gráfica 1 muestra la ecuación y la recta de regresión entre el número de parto de las cerdas y el número de lechones nacidos vivos. Esta gráfica indica que al aumentar el número de partos en la cerda disminuye la cantidad de lechones vivos que puede parir. El coeficiente de determinación señala que el número de partos que tuvieron las cerdas, influyó en forma negativa en un $31 \%$ en la cantidad de lechones nacidos vivos. La gráfica 2 representa en forma de barras la relación entre el número de parto de las cerdas y los lechones nacidos vivos. Aunque se registra alguna variación en la cantidad de lechones que nacen vivos y los partos que ha tenido la cerda, la tendencia es negativa, en particular del séptimo parto en adelante.

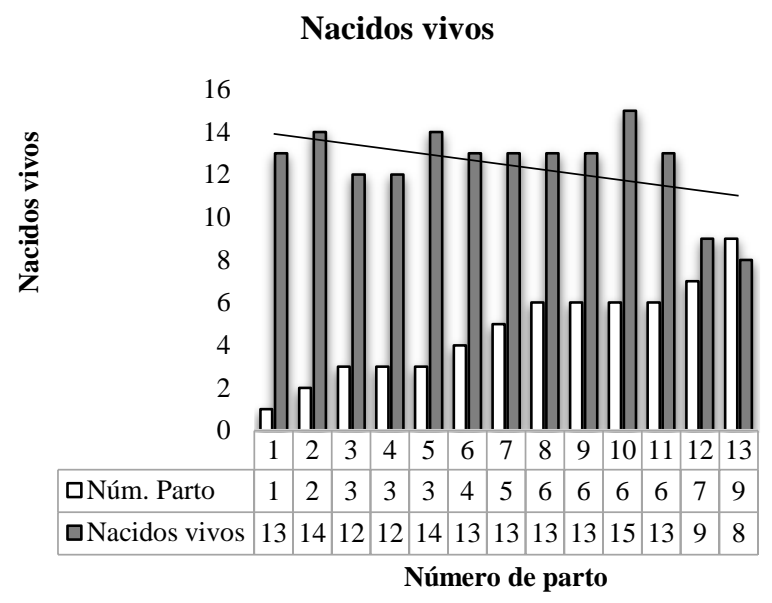

Gráfica 2 Número de parto y lechones nacidos vivos

La gráfica 3 muestra la ecuación y la recta de regresión entre el número de parto de las cerdas y el peso de la camada. La información que proporciona esta figura indica que al aumentar el número de partos en la cerda disminuye el peso de los lechones al nacer.
El coeficiente de determinación señala que el $44 \%$ del peso de las camadas recibieron un efecto descendente según los partos que ha tenido la hembra.

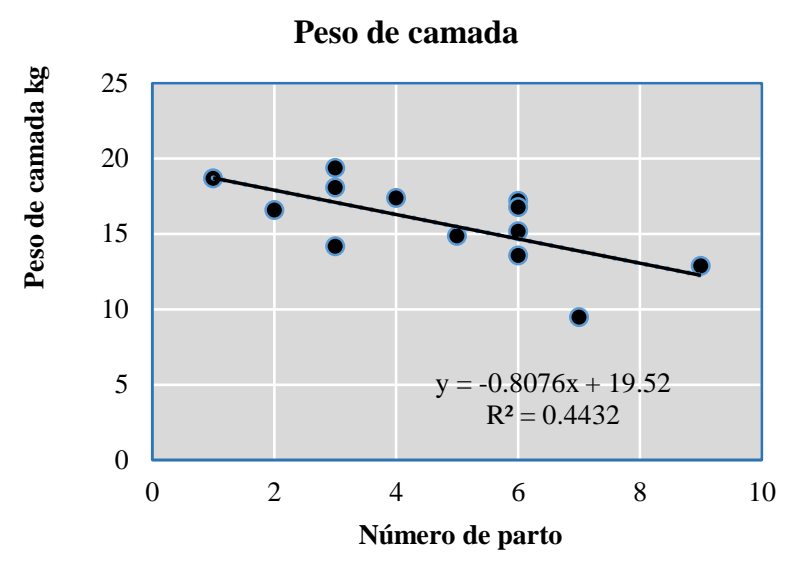

Gráfica 3 Regresión entre número de parto y peso de camada

La gráfica 4 muestra cómo se relaciona el número de parto de las cerdas y el peso de las camadas. La tendencia descendente al aumentar el número de partos se evidencia en esta gráfica, como se observa en la línea de regresión. Excepto la camada 10, del quinto parto en adelante disminuye el peso de la camada.

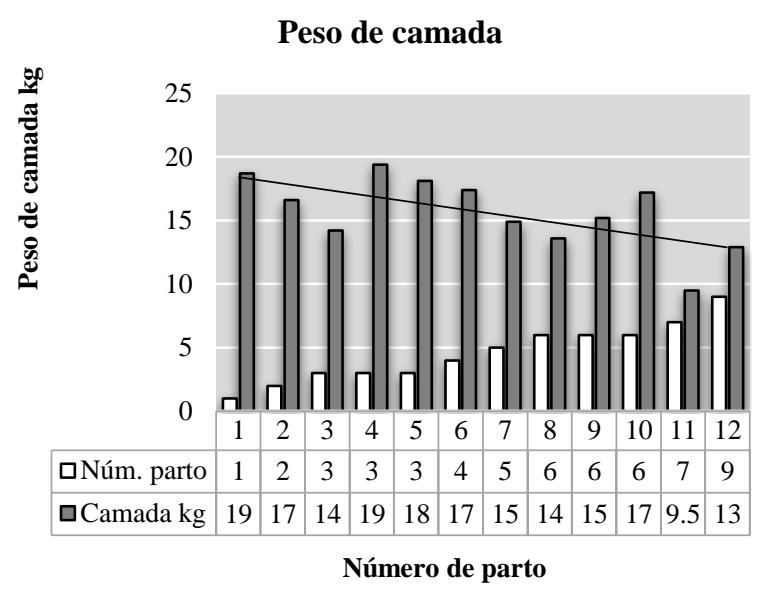

Gráfica 4 Número de parto y peso de camada

La gráfica 5 muestra la ecuación y la recta de regresión entre peso de las camadas y el respectivo peso al destete. La influencia del peso de la camada al nacer es positiva en el peso de los lechones al destete, aunque el coeficiente de determinación sea bajo. 


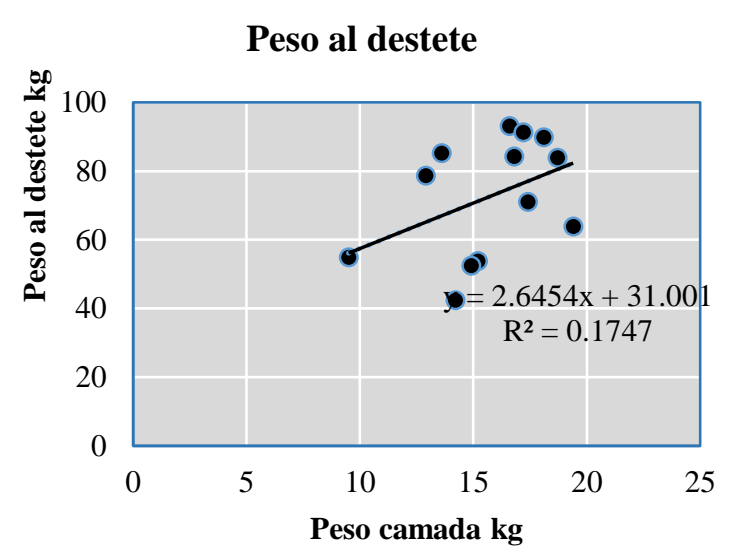

Gráfica 5 Regresión entre peso de camada y peso al destete

La gráfica 6 representa visualmente la relación entre el peso de las camadas y el respectivo peso al destete. La variación que se observa entre camadas, en particular las camadas 4, 5, 6 y 13, hace que el coeficiente $\mathrm{R}^{2}$ sea bajo, aunque en términos generales la tendencia es positiva, como se evidencia en los valores ajustados de la recta.

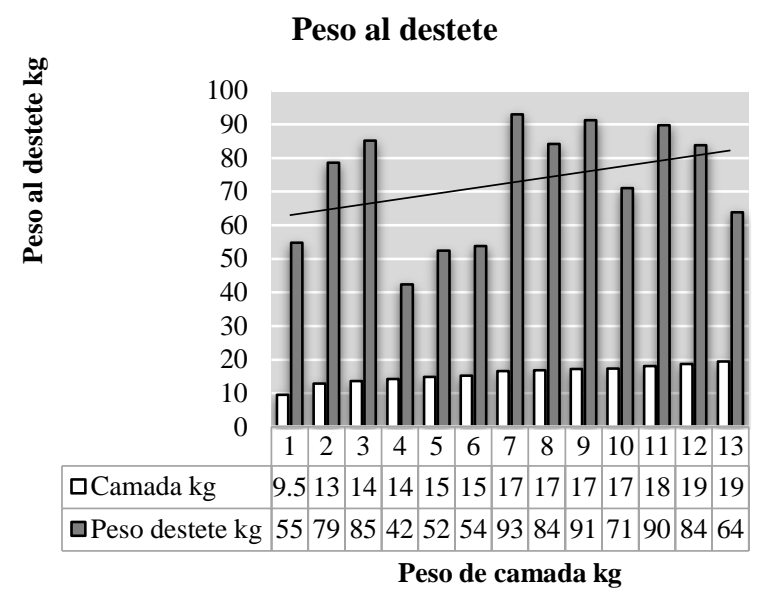

Gráfica 6 Peso de camada y peso al destete

La gráfica 7 contiene la ecuación y la recta de regresión entre el número de lechones nacidos vivos y los lechones destetados. El número de lechones destetados está relacionado en forma positiva con los lechones nacidos vivos, aunque el coeficiente $\mathrm{R}^{2}$ no es fuerte.

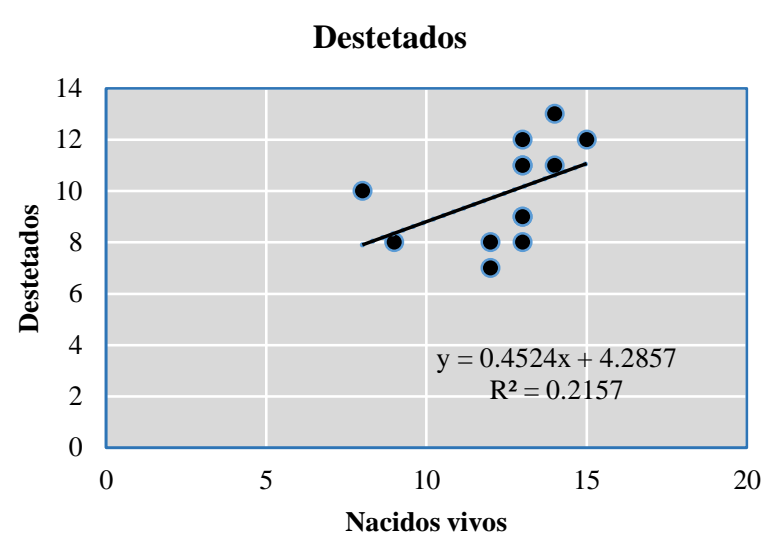

Gráfica 7 Regresión entre lechones nacidos vivos y lechones destetados

En la tabla 2 se anotan las ecuaciones de regresión múltiple con sus respectivos coeficientes de determinación, para ilustrar la interrelación entre aspectos de las cerdas como número de parto, los lechones nacidos vivos, peso individual de los lechones al nacer y los días de lactancia, con el resto de las variables registradas.

\section{Variables}

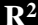

Número de parto con nacidos vivos, nacidos 0.9486

muertos, peso individual, peso camada, mortandad

núm. destetados, peso camada destetada, peso

individual destetados, días lactancia.

$\mathrm{y}=33.828-2.151+0.443+0.744-29.790+2.637+2.555-$ $0.647+7.955-0.238$

Nacidos vivos con peso camada, peso individual, 0.9906

mortandad, núm. lechones destetados, peso camada

destetada, peso individual destetados, días lactancia.

$\mathrm{y}=3.990+0.538-8.182-0.673+0.947-0.193+0.069$

Peso individual lechones al nacer con peso camada, 0.8619

mortandad, núm. lechones destetados, peso camada destetada, peso individual destetados, días lactancia. $\mathrm{y}=-1.624-0.038-0.167+0.390-0.076+0.780-0.006$

Días de lactancia con mortandad, núm. lechones 0.2204

destetados, peso camada destetada, peso individual destetados

$\mathrm{y}=40.371+1.747-0.930+0.319-3.598$

Tabla 2 Regresión múltiple entre número de parto, lechones nacidos vivos, peso individual de lechones al nacer y días de lactancia con variables productivas relacionadas

\section{Discusión}

Considerando la información de todas las cerdas de este estudio, el tamaño de la camada al nacimiento y del peso al nacimiento pueden considerarse relativamente excelentes comparados con los obtenidos en otros estudios (Segura et al., 2007). 
Aunque la diferencia no es marcada, es evidente que a mayor número de parto se reduce el tamaño de camada, en los estudios en que la diferencia a favor de las hembras de dos o más partos ha sido significativa, se ha explicado por el mayor tamaño, capacidad uterina, tasa de ovulación y sobrevivencia embrionaria (Ruiz y Jhonson, 2001). De manera similar (Koketsu et al., 1997), observaron un mejor desempeño reproductivo en cerdas con número de parto igual o mayor que dos comparado con las de primer parto. El PDE fue diferente debido a la cantidad de lechones por cerda, sin embargo, sus pesos le permiten al cerdo ser viable despues de la lactancia, esta condicion de peso puede ser resultado que refleja un manejo y nutrición similar en la lactancia, y la homogenización de las camadas al parto.

Cuando el destete se realiza a una misma edad, el peso de la camada al destete depende principalmente del número de lechones destetados. En un estudio reportado por Ordaz et al. (2013), también se observó que el PCD se incrementó $(\mathrm{p}<0.05)$ conforme el largo de lactancia aumentó, de $40.32 \pm 1.35 \mathrm{~kg}$ con lactancias de 1 a 7 días, hasta $64.01 \pm 0.52 \mathrm{~kg}$ con lactancia iguales o mayores a 29 días.

Los resultados en las correlaciones de acuerdo al número de parto de las cerdas, indicaron la existencia de una correlación alta y positiva con todas las variables de estudio. Resultado similar al obtenido previamente por Murillo et al. (2007) lo que resalta el uso de estas variables como indicadores de la productividad materna de las cerdas la cual incrementa en cada parto.

\section{Referencias}

Batista, L., 2000. Importancia de la evaluación de parámetros reproductivos. V Simposium Internacional de Reproducción e Inseminación Artificial en Porcinos. Ed. Alberto Stephano. 109-114.

Flores, J. J., y Gómez M. A., 1995. Alternativas para el desarrollo de la porcicultura jalisciense. La producción porcícola en México. Contribución al desarrollo de una visión integral. Luis Kato Coordinador. UAM.
Kato, L., 1995. Producción porcícola intensiva. En Kato, L. La producción porcícola en México. Universidad Autónoma Metropolitana. México 30-34.

Koketsu Y, Dial GD, King VL. 1997. Influence of various factors on farrowing rate on farms using early weaning. J Anim Sci ;75:2580-2587. INEGI (Instituto Nacional de Estadística Geografía e Historia). Censo Agropecuario

Murillo, C.; Herradora, M. A.; Martínez, R. 2007. Relación entre la pérdida de grasa dorsal de cerdas lactantes con el consumo de alimento, tamaño de la camada, peso de los lechones al destete y días de lactancia.Rev. Cientif. FCVLUZ. XVII (4): 380-385.

Ordaz-Ochoa, G.; Juárez-Caratachea, A.; García-Valladares, A.; Pérez-Sánchez, R.E.; Ortiz-Rodríguez, R. 2013 Efecto del número de parto sobre los principales indicadores reproductivos de las cerdas. Rev. Cientif. FCVLUZ. XXIII (6):511-519. 2013.

Proyecto de Ordenamiento Territorial del Estado de Jalisco (POET)., 1998. Descripción y diagnostico de la actividad pecuaria en Jalisco. Grupo pecuario. Universidad de Guadalajara. 11-15.

Ruiz-Flores A, Johnson RK. 2001. Direct and correlated responses to two-stage for ovulation rate and number of fully formed

pigs in swine. J Anim Sci ;79:2286-2297

Secretaría de Agricultura y Desarrollo Social (SADER) Servicio de Información Agroalimentaria y Pesquera (SIAP) https://nube.siap.gob.mx/cierre_pecuario/

Segura CJC, Alzina-López A, Solorio RJL. 2007 .Evaluación de tres modelos y factores de riesgo asociados a la mortalidad de lechones al nacimiento en el trópico de México. Tec Pecu Méx ;45:227-236. 